\title{
Traits influencing yield in sesame (Sesamum indicum. L.) and multilocational trials of yield parameters in some desirable plant types
}

\author{
Sandipan Chowdhury ${ }^{1}$, Animesh K. Datta ${ }^{1}$, Aditi Saha ${ }^{2}$, Sonali Sengupta ${ }^{3}$, Rita Paul ${ }^{4}$, Susmita Maity ${ }^{1}$ and Ananya Das ${ }^{1}$ \\ ${ }^{1}$ Dept. of Botany, Genetics and Plant Breeding Section, University of Kalyani, Kalyani - 741235, India \\ ${ }^{2}$ Dept. of Botany, Narasinha Dutt College, Howrah - 711 101, W.B., India \\ ${ }^{3}$ P. G. Dept. of Botany, Hoogly Mohosin College, Hoogly, W.B., India \\ ${ }^{4}$ Dept. of Botany, Charuchandra College, Kolkata - 700 029. W.B., India
}

dattaanimesh@gmail.com

\begin{abstract}
For ascertaining component (s) maximizing yield, 21 genotypes (parental cultivar and 20 macromutants) of sesame (Sesamum indicum. L., var - B-67, Family: Pedaliaceae) were analyzed based on genetic parameters viz. genetic variability, character association and path analysis considering seven yield related traits (plant height, number of primary branches/plant, total branches/plant, distance from base to first branching, capsules on main axis, total capsules/plant and capsule length) and yield (seed yield/seed protein content/seed fatty oil content) in rainfed kharif season of West Bengal plains. Total capsule per plant is predicted to be the most important selection criterion. For assessment of variation in yield attributes, 6 desirable plant types (parental cultivar and five mutants) were given multilocational trial under four different agroclimatic conditions and the results obtained are discussed and the better plant types are predicted.
\end{abstract}

Keywords: Sesamum indicum. L, yield related traits, multilocational trial, agroclimatic conditions.

Introduction

For efficient breeding and crop improvement it is of utmost importance in any crop plant to ascertain the contribution of each yield related trait to yield and to select component maximizing yield. Such studies will also be helpful in determining model plant type for the species. With a view to it, the present investigation is undertaken to study genetic variability, character association and path analysis (basic requirement for yield analysis) in 21 genotypes (normal and 20 mutants) of sesame, Sesamum indicum L. (Family: Pedaliaceae), much valued oil crop with immense therapeutic uses, considering 7 yield related traits (plant height, number of primary branches/plant, total branches/plant, distance from base to first branching, capsules on main axis, total capsules/plant and capsule length) and yield (seed/protein/oil) in rainfed kharif season (best season for sesame cultivation in West Bengal plains). Further, yield (seed/protein/oil) was assessed in parental cultivar and five desirable mutants of sesame (Chowdhury \& Datta, 2008; Chowdhury et al., 2009a, b) under multilocational trials in four different locations (districts) of West Bengal to

\begin{tabular}{|l|c|c|c|c|c|}
\multicolumn{1}{|c}{ Table 1. Estimate of genetic parameters in sesame } \\
\cline { 1 - 4 } Attributes & $\begin{array}{c}\text { Population } \\
\text { mean } \pm \text { S. E. }\end{array}$ & $\begin{array}{c}\text { Coefficient } \\
\text { of variability } \\
(\%)\end{array}$ & $\begin{array}{c}\text { Heritability } \\
(\%)\end{array}$ & $\begin{array}{c}\text { Genetic } \\
\text { gain as } \\
\% \text { of mean }\end{array}$ & Attributes \\
\cline { 2 - 5 } & GCV & PCV & 38.53 & 98.61 & 78.27 \\
\hline Plant height (cm) & $91.08 \pm 4.35$ & 38.26 & 43.98 & 89.03 & 80.66 \\
\hline Primary branches / plant & $3.77 \pm 0.21$ & 41.50 & 51.02 & 97.54 & 102.52 \\
\hline Total branches/ plant & $6.99 \pm 0.44$ & 50.39 & 57.16 & 97.70 & 115.05 \\
\hline $\begin{array}{l}\text { Distance from base to } \\
\text { first branching(cm) }\end{array}$ & $26.69 \pm 1.89$ & 56.50 & & & 81.77 \\
\hline Capsule on main axis & $24.30 \pm 1.24$ & 40.38 & 41.08 & 96.63 & 81.78 \\
\hline Total capsules / plant & $48.00 \pm 2.72$ & 45.05 & 45.56 & 97.79 & 91.78 \\
\hline Capsule length (cm) & $2.24 \pm 0.04$ & 15.54 & 15.98 & 94.61 & 31.14 \\
\hline Seed yield (gm) & $5.77 \pm 0.28$ & 39.02 & 39.62 & 96.97 & 79.16 \\
\hline Seed protein content (\%) & $14.47 \pm 0.36$ & 19.31 & 19.90 & 94.11 & 38.58 \\
\hline Seed fatty oil content (\%) & $34.10 \pm 0.46$ & 10.25 & 10.76 & 90.64 & 20.10 \\
\hline
\end{tabular}

ascertain the influence of agroclimatic conditions on yield. Apart from being an oil yielding plant species, sesame also possessed many agronomic advantages like, capacity to set seeds under high temperature, a deep tap root system that grows well by intercropping and fits well into crop rotation.

\section{Materials and methods}

The experiment was laid with 21 (parental cultivar and 20 plant type mutants - Chowdhury \& Datta, 2008) genotypes (true breeding) of Sesamum indicum var. B-67 at the experimental plots of Kalyani University during May to August 2008 (rainfed kharif season) in design described earlier (Chowdhury et al., 2009a, b). Data were assessed for 7 yield related traits (Table 1) and yield 
(seed/protein/oil) from randomly selected 5 plants from each replications for studying genetic variability, character association and path analysis as per method described by Burton (1952), Johnson et al. (1995) and Dewey and Lu (1959) respectively.

Some desirable plant types (parental cultivar; mutants-elongated leaf, thick leaf, ovate leaf, bushy and unbranched stem) were assessed for seed yield and seed protein and fatty oil (estimated as per method described earlier by Chowdhury et al., 2009a, b) content (following the use of Duncan's t-test analysis, alphabets denotes degree of significance-a>b>c>d>e>f>g ) in agronomically four different locations (districts) of West Bengal (NadiaKalyani University experimental field: Latitude $22^{\circ} 50^{\prime}$ to $24^{\circ} 11^{\prime} \mathrm{N}$, longitude $88^{\circ} 09^{\prime}$ to $88^{\circ} 48^{\prime} \mathrm{E}$; North 24 Parganas - Dattapukur farmer field: Latitude $22^{\circ} 11^{\prime} 6$ ' to $23^{\circ} 15^{\prime 2}{ }^{\prime \prime} \mathrm{N}$, longitude $88^{\circ} 20^{\prime}$ to $89^{\circ} 5^{\prime} \mathrm{E}$; Burdwan Shamsyar farmer field: Latitude $23^{\circ} 15^{\prime}$ to $23^{\circ} 25^{\prime} \mathrm{N}$, longitude $87^{\circ} 45^{\prime}$ to $87^{\circ} 75^{\prime} \mathrm{E}$; Birbhum - Rampurhat farmer's field: Latitude $23^{\circ} 32^{\prime} 30^{\prime \prime}$ to $24^{\circ} 35^{\prime} \mathrm{N}$, Longitude $88^{\circ} 1^{\prime} 40^{\prime \prime}$ to $87^{\circ} 5^{\prime} 25^{\prime \prime} \mathrm{E}$ ). Chemical (carbon, hydrogen and nitrogen contents analyzed by $\mathrm{CHN}$ analyzer; micronutrients estimated from Atomic Absorption Spectroscopy) and physical (sand, silt and clay contents and texture) contents of soil samples from four locations were reported (Chowdhury et al., 2009b) earlier (analysis done in Marine Wing, GSI, Kolkata, West Bengal). Agrometeorological (May to August) data (Nadia: temperature- $31.6^{\circ} \mathrm{C}$ to $39.7^{\circ} \mathrm{C}$ max., $24.0^{\circ} \mathrm{C}$ to $26.8^{\circ} \mathrm{C}$ min., relative humidity $-92.2 \%$ to $99.2 \%$ max., $56.8 \%$ to $84.7 \%$ min., rainfall $-2.08 \mathrm{~mm}$ to $18.18 \mathrm{~mm}$; North 24
Parganas: temperature $-30.2^{\circ} \mathrm{C}$ to $40.2^{\circ} \mathrm{C}$ max., $23.6^{\circ} \mathrm{C}$ to $27.2^{\circ} \mathrm{C}$ min., relative humidity $-94.4 \%$ to $98.2 \%$ max., $51.2 \%$ to $79.4 \%$ min., rainfall $-1.62 \mathrm{~mm}$ to $21.4 \mathrm{~mm}$; Burdwan: temperature $-28.0^{\circ} \mathrm{C}$ to $41.2^{\circ} \mathrm{C}$ max., $22.8^{\circ} \mathrm{C}$ to $27.6^{\circ} \mathrm{C}$ min., relative humidity $-92.9 \%$ to $98.8 \%$ max., $57.2 \%$ to $74.4 \%$ min., rainfall $-2.52 \mathrm{~mm}$ to $20.8 \mathrm{~mm}$; Birbhum: temperature $-34.0^{\circ} \mathrm{C}$ to $44.2^{\circ} \mathrm{C}$ max., $25.2^{\circ} \mathrm{C}$ to $27.6^{\circ} \mathrm{C}$ min., relative humidity $-88.8 \%$ to $98.6 \%$ max., $56.4 \%$ to $75.2 \%$ min., rainfall $-1.02 \mathrm{~mm}$ to $10.6 \mathrm{~mm}$ ) were obtained from Agrometeorological Department of B.C.K.V. , Mohanpur and Govt. of West Bengal, Writer's Building, Kolkata.

\section{Results and discussion}

Estimates of genetic parameters (Table 1) indicated that the magnitude of GCV (genotypic coefficient of variation) and $\mathrm{PCV}$ (phenotypic coefficient of variation) were low to moderate, and for all the traits PCV and GCV values were more or less similar. Heritability (broad sense) for different quantitative traits was high $(89.03 \%$ to $98.61 \%$ ) and it was coupled with high genetic gain (index for selection criteria) excepting for capsule length and seed protein and fatty oil contents.

Correlation studies (Table 2) showed that for all characters genotypic and phenotypic associations were in the same direction and genotypic estimates were relatively higher than the phenotypic ones indicating an inherited association between the traits. Capsule on the main axis and total capsules per plant were positively and significantly $(p>0.01)$ correlated with seed yield and seed protein content. Seed yield per plant was also significantly $(p>0.05)$ associated with seed protein Table 2. Phenotypic $(P)$ and Genotypic $(G)$ correlation between different attributes and between different traits with seed oil content

\begin{tabular}{|c|c|c|c|c|c|c|c|c|c|c|c|}
\hline Attributes & & $\begin{array}{l}\text { Plant } \\
\text { ht. } \\
(\mathrm{cm})\end{array}$ & $\begin{array}{l}\text { Primary } \\
\text { branch / } \\
\text { plant }\end{array}$ & $\begin{array}{l}\text { Total } \\
\text { branches/ } \\
\text { plant }\end{array}$ & $\begin{array}{l}\text { Distance } \\
\text { from base } \\
\text { to first / } \\
\text { branching } \\
\text { (cm) }\end{array}$ & $\begin{array}{c}\text { Capsule } \\
\text { on Main } \\
\text { axis }\end{array}$ & $\begin{array}{l}\text { Capsule } \\
\text { per plant }\end{array}$ & $\begin{array}{c}\text { Capsule } \\
\text { length } \\
(\mathrm{cm})\end{array}$ & $\begin{array}{l}\text { Seed } \\
\text { yield } \\
(\mathrm{gm})\end{array}$ & $\begin{array}{l}\text { Protein } \\
\text { content } \\
\quad(\%)\end{array}$ & $\begin{array}{l}\text { Oil } \\
\text { content } \\
(\%)\end{array}$ \\
\hline \multirow{2}{*}{$\begin{array}{l}\text { Plant height } \\
\text { (cm) }\end{array}$} & $\mathrm{P}$ & & 0.67 & 0.68 & 0.87 & 0.32 & 0.48 & 0.23 & 0.25 & 0.21 & -0.15 \\
\hline & $\mathrm{G}$ & & 0.69 & 0.69 & 0.88 & 0.32 & 0.49 & 0.24 & 0.26 & 0.21 & -0.16 \\
\hline \multirow{2}{*}{$\begin{array}{l}\text { Primary branch } \\
\text { per plant }\end{array}$} & $\mathrm{P}$ & & & 0.88 & 0.66 & 0.05 & 0.56 & 0.12 & 0.15 & 0.05 & 0.16 \\
\hline & $\mathrm{G}$ & & & 0.92 & 0.70 & 0.07 & 0.59 & 0.11 & 0.15 & 0.05 & 0.15 \\
\hline \multirow{2}{*}{$\begin{array}{l}\text { Total branches } \\
\text { per plant }\end{array}$} & $\mathrm{P}$ & & & & 0.66 & 0.04 & 0.58 & 0.29 & 0.20 & 0.02 & 0.08 \\
\hline & $\mathrm{G}$ & & & & 0.67 & 0.04 & 0.59 & 0.30 & 0.20 & 0.02 & 0.08 \\
\hline \multirow{2}{*}{$\begin{array}{l}\text { Distance from } \\
\text { base to first } \\
\text { branching }(\mathrm{cm})\end{array}$} & $\mathrm{P}$ & & & & & 0.21 & 0.58 & 0.02 & 0.17 & 0.39 & -0.19 \\
\hline & $\mathrm{G}$ & & & & & 0.20 & 0.59 & 0.02 & 0.17 & 0.40 & -0.20 \\
\hline \multirow{2}{*}{$\begin{array}{l}\text { Capsule on } \\
\text { main axis }\end{array}$} & $\mathrm{P}$ & & & & & & 0.52 & 0.35 & 0.74 & 0.56 & 0.04 \\
\hline & $\mathrm{G}$ & & & & & & 0.59 & 0.37 & 0.77 & 0.59 & 0.04 \\
\hline \multirow{2}{*}{$\begin{array}{l}\text { Total capsule } \\
\text { per plant }\end{array}$} & $\mathrm{P}$ & & & & & & & 0.10 & 0.68 & 0.53 & 0.30 \\
\hline & $\mathrm{G}$ & & & & & & & 0.11 & 0.68 & 0.56 & 0.30 \\
\hline \multirow{2}{*}{$\begin{array}{l}\text { Capsule } \\
\text { length }(\mathrm{cm})\end{array}$} & $\mathrm{P}$ & & & & & & & & 0.32 & 0.08 & 0.06 \\
\hline & $\mathrm{G}$ & & & & & & & & 0.33 & 0.08 & 0.06 \\
\hline \multirow{2}{*}{$\begin{array}{l}\text { Seed yield } \\
\text { (gm) }\end{array}$} & $\mathrm{P}$ & & & & & & & & & 0.41 & 0.29 \\
\hline & $\mathrm{G}$ & & & & & & & & & 0.44 & 0.28 \\
\hline \multirow{2}{*}{$\begin{array}{l}\text { Seed } \\
\text { Protein } \\
\text { content (\%) }\end{array}$} & $\mathrm{P}$ & & & & & & & & & & -0.19 \\
\hline & $G$ & & & & & & & & & & -0.19 \\
\hline
\end{tabular}

Significant at $5 \%>0.43 \quad$ Bold values are significant Significant at 1\% $>0.55$

Research article "Sesame"

CIndian Society for Education and Environment (iSee) http://www.indjst.org
Sandipan et al. Indian J.Sci.Technol. 
content but none of the parameters analyzed was significantly interrelated with fatty oil content. Different agroclimatic factor(s) on yield attributes of sesame. Result (Table 5) indicated that seed yield was high in

Table 3. Direct and indirect effects of yield components of sesame on yield of oil content.

\begin{tabular}{|c|c|c|c|c|c|c|c|c|c|c|c|}
\hline Attributes & & $\begin{array}{c}\text { Plant } \\
\text { height } \\
(\mathrm{cm})\end{array}$ & $\begin{array}{c}\text { No of } \\
\text { primary } \\
\text { branch } \\
\text { /plant }\end{array}$ & $\begin{array}{c}\text { Total } \\
\text { Branches } \\
\text { /plant }\end{array}$ & $\begin{array}{l}\text { Distance } \\
\text { from base } \\
(\mathrm{cm}) \text { to } \\
\text { first } \\
\text { branching }\end{array}$ & $\begin{array}{c}\text { Capsule } \\
\text { on the } \\
\text { main } \\
\text { axis }\end{array}$ & $\begin{array}{c}\text { Total } \\
\text { capsule } \\
\text { per plant }\end{array}$ & $\begin{array}{c}\text { Capsule } \\
\text { length } \\
(\mathrm{cm})\end{array}$ & $\begin{array}{l}\text { Seed } \\
\text { yield } \\
(\mathrm{gm})\end{array}$ & $\begin{array}{l}\text { Seed } \\
\text { Protein } \\
\text { content } \\
(\%)\end{array}$ & $\begin{array}{l}\text { Genetic } \\
\text { correlation } \\
\text { with seed } \\
\text { oil content }\end{array}$ \\
\hline $\begin{array}{l}\text { Plant } \\
\text { Height }(\mathrm{cm})\end{array}$ & $\mathrm{K}$ & 0.0326 & 0.0449 & -0.2624 & -0.3546 & -0.0590 & 0.5343 & 0.0103 & -0.0040 & -0.1123 & -0.162 \\
\hline $\begin{array}{l}\text { No. of primary } \\
\text { branch/ plant }\end{array}$ & $\mathrm{K}$ & 0.0225 & 0.0651 & -0.3512 & -0.2795 & 0.0119 & 0.6466 & 0.0049 & -0.0023 & 0.0262 & 0.152 \\
\hline $\begin{array}{l}\text { Total branches } \\
\text { / plant }\end{array}$ & $\mathrm{K}$ & 0.0224 & 0.0598 & -0.3826 & -0.2687 & -0.0075 & 0.6389 & 0.0129 & -0.0030 & -0.0128 & 0.075 \\
\hline $\begin{array}{l}\text { Distance from } \\
\text { base to first } \\
\text { branching }(\mathrm{cm})\end{array}$ & $\mathrm{K}$ & 0.0288 & 0.0453 & -0.2559 & -0.4016 & 0.0367 & 0.6422 & -0.0008 & -0.0027 & -0.2154 & -0.197 \\
\hline $\begin{array}{l}\text { Capsule } \\
\text { on main axis }\end{array}$ & $\mathrm{K}$ & 0.0105 & -0.0042 & -0.0157 & -0.0803 & -0.1832 & 0.5823 & 0.0160 & -0.0017 & -0.3148 & 0.035 \\
\hline $\begin{array}{l}\text { Total capsule/ } \\
\text { plant }\end{array}$ & $\mathrm{K}$ & 0.0160 & 0.0386 & -0.2242 & -0.2365 & -0.0978 & 1.0904 & 0.0046 & -0.0104 & -0.2967 & 0.304 \\
\hline $\begin{array}{l}\text { Capsule } \\
\text { length }(\mathrm{cm})\end{array}$ & $\mathrm{K}$ & 0.0077 & 0.0073 & -0.1129 & 0.0076 & -0.0671 & 0.1145 & 0.0437 & -0.0051 & 0.0417 & 0.063 \\
\hline Seed yield (gm) & $\mathrm{K}$ & 0.0086 & 0.0096 & -0.0761 & -0.0699 & -0.1407 & 0.7425 & 0.0146 & -0.0152 & -0.2347 & 0.281 \\
\hline $\begin{array}{l}\text { Seed protein } \\
\text { content }\end{array}$ & $\mathrm{K}$ & 0.0069 & -0.0032 & -0.0092 & -0.1619 & -0.1079 & 0.6051 & -0.0034 & -0.0067 & -0.5345 & -0.193 \\
\hline
\end{tabular}

Residual effect $(K)-0.7174 ; K=$ Kharif

yield related traits showed positive and significant (probability level 0.05 to 0.01 ) relationship among themselves.

In addition to the degree of associations, path coefficient analysis takes into account the cause and effect relationship and has been performed to partition the genetic correlation into direct and indirect effects for understanding the relative importance of the component characters on yield (traits analyzed for oil yield). Results (Table 3 ) obtained for path analysis revealed that only total capsules per plant (1.0904) had substantial positive and direct contribution to oil yield; while, the other traits showed poor or negative contribution. Significance of total capsule per plant as important selection criteria in sesame was also suggested earlier (Mishra et al., 1993; Biswas and Akbar, 1995; Singh et al., 1997; Sengupta and Datta, 2004). However, high residual effect (0.7174) in path analysis suggested the inclusion of other traits for better precision.

Pooled ANOVA (Table 4) performed over the districts for 3 yield parameters considering six plant types (among which parental cultivar, thick leaf, bushy and unbranched stem were better parents as evidenced from Principal Component Analysis and Hierarchial clustering using single linkage and complete linkage method of Dillon and Goldstain (1984) mentioned earlier by Chowdhury (2009) revealed that yield varied significantly among districts and plant types. Further, district $x$ replication (seed yield) and districts $\mathrm{x}$ plant types (protein and oil content) also varied significantly thereby suggesting the influence of bushy mutant in Nadia compared to other districts; while, seed protein content was maximum in thick leaf in Burdwan. Seed protein content varied considerably among districts in unbranched stem mutant but fatty oil content was highest in the plant type. Nadia seems to give better result for the traits analyzed. Over the districts the plant types were in the following order for different traits: seed yield - bushy $>$ thick leaf $>$ elongated leaf $=$ control = unbranched stem $>$ ovate leaf, seed protein content - thick leaf > elongated leaf > ovate leaf > unbranched stem > bushy > control; fatty oil content bushy > unbranched stem > ovate leaf = control > elongated leaf $>$ thick leaf. Thus, the plant types evolved as the consequence of radiation induced mutation in sesame may be utilized directly (not withstanding the marker traits associated with the mutants - Chowdhury et al., 2009a,b) and/or be exploited through intercrossing (between/among) followed by selection.

\section{Acknowledgement}

This research is grant aided by UGC.

\section{Reference}

1. Biswas KP and Akbar MA (1995) Genetic variability, correlation and path analysis in sesame (Sesamum indicum L.). Bangladesh J. Sci. Ind. Res. 30, 71-79.

2. Burton GW (1952) Quantitative inheritance of grass. Proc. $6^{\text {th }}$ Int. Grassland Cong. Held at Pennsylvania State College, Pa. U.S.A, 174-183.
Research article

(C)Indian Society for Education and Environment (iSee)
"Sesame"

http://www.indjst.org
Sandipan et al. Indian J.Sci.Technol. 
Table 4. ANOVA - Pooled over the Districts

\begin{tabular}{|c|c|c|c|c|c|c|c|c|c|c|c|c|}
\hline \multirow{2}{*}{ Source } & \multicolumn{4}{|c|}{ Seed yield } & \multicolumn{4}{|c|}{ Seed protein } & \multicolumn{4}{|c|}{ Oil } \\
\hline & SS & $\mathrm{df}$ & MSS & $\mathrm{F}$ & SS & df & MSS & $\mathrm{F}$ & SS & df & MSS & $\mathrm{F}$ \\
\hline District & 4.08 & 3 & 1.36 & $3.58^{*}$ & 58.57 & 3 & 19.52 & $44.36^{* \star *}$ & 517.85 & 3 & 172.33 & $152.50^{* * \star}$ \\
\hline District X Replication & 17.01 & 6 & 2.84 & $7.47^{\star \star \star}$ & 2.94 & 6 & 0.49 & 1.11 & 4.42 & 6 & 0.74 & 0.65 \\
\hline District X Plant types & 5.62 & 15 & 0.37 & 1.00 & 111.14 & 15 & 7.41 & $16.84^{* \star *}$ & 125.91 & 15 & 8.39 & $7.42^{* * *}$ \\
\hline Error (District) & 11.29 & 30 & 0.38 & & 13.26 & 30 & 0.44 & & 34.01 & 30 & 1.13 & \\
\hline Replication & 0.22 & 2 & 0.11 & 0.92 & 0.38 & 2 & 0.19 & 1.12 & 1.21 & 2 & 0.60 & 0.34 \\
\hline Plant type & 23.13 & 5 & 4.63 & $38.58^{* \star *}$ & 655.46 & 5 & 131.09 & $771.11^{\star \star *}$ & 1154.56 & 5 & 230.91 & $129.72^{\star * \star}$ \\
\hline Error & 1.17 & 10 & 0.12 & & 1.71 & 10 & 0.17 & & 17.76 & 10 & 1.78 & \\
\hline
\end{tabular}

3. Chowdhury S and Datta AK (2008) Radiation induced macromutation in sesame (Sesamum indicum L.). J. Phytol. Res. 21 (2), 181-185.

4. Chowdhury $S$ (2009) Radiation Induced Mutagenesis In Sesame (Sesamum indicum L.). Ph.D Thesis. Univ. of Kalyani.

5. Chowdhury S, Datta AK and Maity S (2009a) Cytogenetical and agronomical aspects of radiation induced marker trait mutants in sesame (Sesamum indicum L.). Indian J. Sci. Technol. 2 (5\&6), 58-61.

6. Chowdhury S, Datta AK, Saha A and Maity S (2009b) Radiation induced two oil rich mutants in sesame (Sesamum indicum L.). Indian J. Sci. Technol. 2 (7), 51-52.

7. Dewey JR and Lu KH (1959) A correlation and path coefficient analysis of components of crested white grass seed production. Agron. J. 47, 477-483.

8. Dillon WR and Goldstein M (1984) Multivariate analysis methods and application. John Wiley and Sons (eds.), pp, 209-242.

9. Johnson HW, Robinson HF and Comstock RE (1995) Estimates of genetic and environmental variability in soybean. Agron. J. 47, 314-318.

10. Mishra AK, Ali SA, Rai HS, Ghurayya RS and Yadav LN (1993) Genetic variability, correlation and path analysis in sesame. Int. Trop. Agric. 11, 113-117.
11. Sengupta S and Datta AK (2004) Genetic studies to ascertain selection criteria for yield improvement in sesame. J. Phytol. Res. 17, 163-166.

12. Singh RM, Singh AK, Kumar $P$, Thakral NK and Kumar $P$ (1997) Association of yield and its component traits in sesame. Ann. Biol. Ludhiana. 13, 47-51.

Table 5. Seed yield and protein and oil contents in sesame plant types in four different locations of West Bengal

\begin{tabular}{|c|c|c|c|c|c|c|c|c|c|c|c|c|c|c|c|c|}
\hline \multirow[b]{2}{*}{$\begin{array}{l}\text { Plant } \\
\text { types }\end{array}$} & & \multicolumn{5}{|c|}{ Seed yield $(\mathrm{gm})$} & \multicolumn{5}{|c|}{ Seed protein content (\%) } & \multicolumn{5}{|c|}{ Seed oil content (\%) } \\
\hline & & 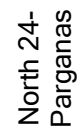 & 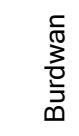 & 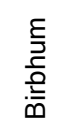 & $\frac{\frac{\pi}{7}}{\frac{\pi}{Z}}$ & $\begin{array}{l}\overline{\bar{\pi}} \\
\frac{\pi}{0} \\
0\end{array}$ & 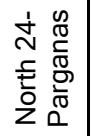 & $\begin{array}{l}\frac{5}{\pi} \\
30 \\
\frac{3}{3} \\
0\end{array}$ & $\begin{array}{l}\frac{5}{5} \\
\frac{2}{0} \\
\frac{2}{0} \\
0\end{array}$ & $\frac{.0}{\frac{\pi}{\pi}}$ & $\begin{array}{l}\overline{\bar{\sigma}} \\
\bar{\phi} \\
\bar{\partial}\end{array}$ & 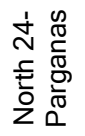 & $\begin{array}{l}\frac{1}{00} \\
\frac{0}{3} \\
\frac{0}{3} \\
0\end{array}$ & 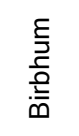 & $\frac{\frac{\pi}{0}}{\frac{\pi}{2}}$ & $\begin{array}{l}\overline{\bar{\sigma}} \\
\overline{0} \\
0\end{array}$ \\
\hline \multirow{2}{*}{ Control } & ean & 6.29 & 6.37 & 5.99 & 6.37 & $6.26 \mathrm{~d}$ & 12.00 & 13.90 & 13.13 & 12.83 & $12.97 \mathrm{f}$ & 29.93 & 31.43 & 26.13 & 34.35 & $30.40 d$ \\
\hline & S.E. & 0.23 & 0.18 & 0.20 & 0.16 & 0.19 & 0.23 & 0.17 & 0.34 & 0.19 & 0.23 & 0.18 & 0.69 & 1.30 & 0.81 & 0.74 \\
\hline $\begin{array}{l}\text { Elongate } \\
\text { leaf }\end{array}$ & Mean & 6.31 & 6.07 & 6.53 & 7.25 & $6.54 d$ & 14.87 & 17.27 & 16.27 & 16.63 & $16.26 b$ & 29.40 & 31.40 & 26.40 & 33.40 & $30.10 \mathrm{~d}$ \\
\hline \multirow{2}{*}{$\begin{array}{l}\text { Thick } \\
\text { leaf }\end{array}$} & Mean & 7.97 & 7.77 & 7.47 & 7.83 & $7.76 \mathrm{c}$ & 19.43 & 21.97 & 19.13 & 21.40 & $20.48 a$ & 26.07 & 26.07 & 22.20 & 27.57 & $25.40 \mathrm{e}$ \\
\hline & S.E. & 0.23 & 0.24 & 0.15 & 0.14 & 0.19 & 0.19 & 0.67 & 0.18 & 0.64 & 0.42 & 0.35 & 0.18 & 0.23 & 0.35 & 0.28 \\
\hline \multirow{2}{*}{$\begin{array}{l}\text { Ovate } \\
\text { leaf }\end{array}$} & Mean & 5.08 & 5.31 & 5.17 & 5.20 & $5.19 \mathrm{e}$ & 14.27 & 16.10 & 15.60 & 15.37 & $15.33 c$ & 30.33 & 32.33 & 28.47 & 32.87 & $31.00 \mathrm{~cd}$ \\
\hline & S.E. & 0.19 & 0.14 & 0.18 & 0.02 & 0.13 & 0.18 & 0.15 & 0.12 & 0.56 & 0.25 & 0.59 & 0.18 & 0.58 & 0.75 & 0.53 \\
\hline \multirow{2}{*}{ Bushy } & Mean & 9.28 & 8.55 & 8.62 & 9.49 & $8.99 a$ & 12.73 & 14.33 & 14.23 & 13.83 & $13.78 \mathrm{e}$ & 30.33 & 33.93 & 29.67 & 35.87 & $32.40 \mathrm{~b}$ \\
\hline & S.E. & 0.50 & 0.54 & 0.36 & 0.07 & 0.37 & 0.24 & 0.24 & 0.15 & 0.23 & 0.21 & 0.47 & 0.41 & 0.30 & 0.413 & 0.41 \\
\hline $\begin{array}{l}\text { Unbran } \\
\text {-ched } \\
\text { stem }\end{array}$ & Mean & 6.42 & 6.32 & 6.45 & 6.62 & $6.45 \mathrm{~d}$ & 12.20 & 13.23 & 12.80 & 20.13 & $14.59 \mathrm{~d}$ & 30.07 & 29.60 & 27.27 & 9.43 & $31.50 \mathrm{bc}$ \\
\hline
\end{tabular}

Research article

Clndian Society for Education and Environment (iSee)
"Sesame"

http://www.indjst.org
Sandipan et al. Indian J.Sci.Technol. 